\title{
Pancreas Cancer Incidence and Pancreas Cancer-Associated Mortality Are Low in National Cohort of 7211 Pancreas Cyst Patients
}

\author{
Gobind S. Anand ${ }^{1,2}$ (1) $\cdot$ Fady Youssef ${ }^{2} \cdot$ Lin Liu $^{1,2} \cdot$ Ranier Bustamante $^{1} \cdot$ Ashley Earles $^{1} \cdot$ Santhi Swaroop Vege ${ }^{3} \cdot$ \\ Thomas Savides $^{2}$. Syed Abbas Fehmi ${ }^{2}$. Wilson T. Kwong ${ }^{2}$. Samir Gupta ${ }^{1,2}$
}

Received: 9 October 2020 / Accepted: 23 February 2021 / Published online: 30 March 2021

(c) This is a U.S. government work and not under copyright protection in the U.S.; foreign copyright protection may apply 2021

\begin{abstract}
Background and Aims Pancreatic cancer incidence and mortality among patients with pancreas cysts are unclear. The aims of this study are to evaluate incidence of pancreatic cancer and cause-specific mortality among patients with pancreatic cysts using a large national cohort over a long follow-up period.

Methods We conducted a retrospective cohort study of US Veterans diagnosed with a pancreatic cyst 1999-2013, based on International Classification of Diseases, 9th edition (ICD9) coding within national Department of Veterans Affairs (VA) data. Pancreatic cancer incidence was ascertained using VA cancer registry data, ICD-9 codes, and the National Death Index, a national centralized database of death records, including cause-specific mortality.

Results Among 7211 Veterans with pancreatic cysts contributing 31,501 person-years of follow-up (median follow-up 4.4 years), 79 (1.1\%) developed pancreatic cancer. A total of 1982 patients (27.5\%) died during the study follow-up period. Sixty-three patients (3.2\% of deaths; $0.9 \%$ of pancreas cyst cohort) died from pancreatic cancer, but the leading causes of death in the cohort were non-pancreatic cancer ( $n=498,25 \%$ of deaths) and cardiovascular disease ( $n=398,20 \%$ of deaths). Conclusions Pancreas cancer incidence and pancreatic cancer-associated mortality are very low in a large national cohort of VA pancreatic cyst patients with long-term follow-up. Most deaths were from non-pancreas cancers and cardiovascular causes, and only a minority (3.2\%) were attributable to pancreas cancer. Given death from pancreas cancer is rare, future research should focus on identifying criteria for selecting individuals at high risk for death from pancreatic cancer for pancreatic cyst surveillance.
\end{abstract}

Keywords Pancreas cyst $\cdot$ Pancreas cancer incidence $\cdot$ Mortality $\cdot$ Cohort study

Gobind S. Anand

gsanand@health.ucsd.edu

Fady Youssef

fayoussef@health.ucsd.edu

Lin Liu

12liu@ucsd.edu

Ranier Bustamante

rabustamante@health.ucsd.edu

Ashley Earles

aearles@health.ucsd.edu

Santhi Swaroop Vege

vege.santhi@mayo.edu

Thomas Savides

tsavides@health.ucsd.edu
Syed Abbas Fehmi

sfehmi@health.ucsd.edu

Wilson T. Kwong

wtkwong@health.ucsd.edu

Samir Gupta

s1gupta@health.ucsd.edu

1 Veterans Affairs San Diego Healthcare System, $3350 \mathrm{La}$ Jolla Village Drive MC 111D, San Diego, CA 92161, USA

2 University of California San Diego, 9500 Gilman Drive, La Jolla, CA, USA

3 Mayo Clinic, 200 1st St SW, Rochester, MN, USA 


\section{Introduction}

Pancreatic cysts are commonly seen with an estimated prevalence of 2-15\% [1-6]. Furthermore, with the increasing use of cross-sectional imaging, a greater number of incidental pancreatic cysts are being identified in patients without other signs or symptoms of pancreatic disease [7]. Once detected, these cysts can trigger significant anxiety about developing and dying from pancreatic cancer. Pancreatic cysts encompass benign cystic lesions such as pseudocysts and serous cystadenomas and potentially malignant cysts such as intraductal papillary mucinous neoplasms (IPMNs) and mucinous cystic neoplasms (MCNs). However, our ability to accurately determine cyst type, risk for developing cancer, and need for surgery is limited. Further, surgery for pancreatic cysts is associated with significant mortality and morbidity [8].

The incidence of pancreatic cancer among patients with incidentally found pancreatic cysts is postulated to be low but has not been well characterized. Most of the data regarding incidence of pancreatic cancer among patients with pancreatic cysts are based on surgical resection data. The major challenges to apply findings from surgical series include inherent problems with (a) selection bias and (b) an unclear understanding about the long-term outcomes in patients with non-malignant cysts at time of surgery (i.e., would some of these cysts eventually develop cancer?). Published non-surgical series are limited by small size, small numbers of patients developing pancreatic cancer and short-term follow-up resulting in imprecise estimates of pancreatic cancer incidence.

Even less is known about pancreas cancer-associated mortality among patients with pancreatic cysts due to a dearth of population-based studies evaluating the natural history of pancreatic cysts. Additional work is needed to clarify the pancreatic cancer-associated mortality after pancreatic cyst diagnosis and place risk of pancreatic cancer mortality in context of competing mortality risks from other causes.

Thus, the primary aim of this study is to calculate incidence of pancreatic cancer among patients with pancreatic cysts using a large national cohort over a long follow-up period. The secondary aim is to evaluate all-cause mortality and cause-specific mortality, including pancreas cancer-associated mortality among patients with pancreatic cysts.

\section{Methods}

\section{Study Design}

We conducted a retrospective cohort study of US Veterans with pancreatic cysts utilizing national Department of Veterans Affairs (VA) electronic health record data accessed through the VA Informatics and Computing Infrastructure (VINCI). VINCI allows secure access to the national VA corporate data warehouse (CDW) for research purposes. CDW data include discrete coded fields, such as prior diagnoses, medication history, clinical laboratories, and demographic characteristics, as well as free-text notes for all Veteran healthcare encounters, such as endoscopic ultrasound reports, clinical progress notes, radiology reports, and pathology reports. Furthermore, the complete electronic medical record of any Veteran can be reviewed via VINCI.

\section{Pancreas Cyst Cohort}

Our pancreas cyst cohort was created by identifying all adult (age $\geq 18$ years) VA patients from January 1, 1999, to December 31, 2013, with International Classification of Diseases, Ninth Revision (ICD-9) code 577.2. Because the 577.2 code is assigned to individuals with pancreatic cyst or pseudocysts, individuals with a concurrent or prior ICD-9 codes for 577.0 (acute pancreatitis) and 577.1 (chronic pancreatitis) were excluded to minimize patients with pancreatic pseudocysts and to avoid patients with potentially symptomatic pancreatic cystic lesions causing pancreatitis. Patients diagnosed with pancreatic cancer within the first year of pancreas cyst diagnosis were excluded to minimize misclassification of patients who had prevalent cancer at time of cyst diagnosis. Patients with shorter than 1 year of follow-up were also excluded.

\section{Pancreas Cancer Ascertainment}

Pancreas cancer was ascertained based on meeting one or more of the following criteria: (1) presence of ICD-9 code $157.0,157.1,157.2,157.3,157.8$, or 157.9 for pancreas cancer; (2) death from pancreas cancer based on the National Death Index (NDI); and (3) presence of an International Classification of Diseases for Oncology, Third Edition (ICDO-3) code for pancreas cancer within the Oncology Domain. The NDI is a centralized database of death records which also contains information on cause-specific mortality and is considered the most accurate death record in the USA [9]. A major advantage of utilizing NDI data is to obtain information for Veterans who obtained care outside of the 
VA after cancer diagnosis. The Oncology Domain within VINCI contains data abstracted from local VA hospital cancer registrars. As part of the current study, manual chart review was performed for all patients with pancreatic cancer to confirm diagnoses. Patients who did not meet all inclusion criteria on chart review were excluded. Patients identified as having pathology other than adenocarcinoma (such as neuroendocrine tumors) were excluded from the analysis. For patients who were only identified utilizing NDI, the date of death from pancreas cancer was used for pancreas cancer diagnosis date. For three patients who were identified by ICD-9 code only, the diagnosis of pancreas cancer was based on high clinical suspicion by the treating physicians as a biopsy was never pursued/performed. These patients were included in the analysis because pancreas cancer is often found in an advanced state clinically, and biopsy is sometimes not pursued based on clinical considerations such as patient preferences.

\section{All-Cause and Cause-Specific Mortality}

All-cause and cause-specific mortality was ascertained using NDI. Cause of death was divided into the following categories: pancreas cancer, non-pancreas cancer, cardiovascular, respiratory, cerebrovascular, hepatic, diabetes, kidney, infection, and other.

\section{Covariates}

Covariates (at time of pancreas cyst diagnosis) including age, gender, race/ethnicity, body mass index (BMI), tobacco use, and Charlson Comorbidity Index Score (CCIS) were ascertained using data within the CDW. Diabetes was based on presence of at least one inpatient ICD-9 diagnosis of diabetes, at least 2 outpatient ICD-9 diagnoses of diabetes, at least 2 prescription fills of diabetes medications of 30 days or greater, or prescriptions for metformin and glucose test strips within a two-year timeframe [10].

\section{Time to Cancer Analysis}

The primary outcome was pancreas cancer diagnosis, defined by date of pancreas cancer diagnosis (based on ICD-9 code date, NDI pancreas cancer death date, or Oncology Domain ICD-O-3 date). For patients diagnosed as having pancreas cancer using multiple methods, the earliest date was utilized. Follow-up time began at time of pancreas cyst diagnosis, which was based on first ICD-9 code for pancreas cyst. For patients who did not develop pancreas cancer, they were censored in the following order: (1) date of pancreatic cyst surgery (based on Current Procedural Terminology codes 48,120, 48,140, 48,145, 48,150, 48,152, 48,153, $48,154)$; (2) date of death during follow-up; (3) date of last VA healthcare encounter; or (4) end of study period (12/31/2014). Date of pancreatic surgery was used to censor because the natural history of the underlying cyst is altered among pancreas cyst patients undergoing surgical resection. If pancreas cancer was present at time of surgery, patients were included in the analysis.

Pancreatic cancer incidence was computed in two ways: (a) using person-years follow-up approach; (b) 5-year, 10-year, and 15-year absolute incidence of pancreatic cancer. Continuous variables were compared using Wilcoxon rank sum test, and categorical variables were compared using Chi-square test between patients with pancreatic cancer and those without pancreatic cancer. Time to pancreatic cancer was estimated using Kaplan-Meier method. Univariable Cox proportional hazards regression was used to study the association between each baseline characteristic and time to pancreatic cancer. These variables were included as predictors in the multivariable Cox regression model if found moderately associated $(p<0.15)$ with the outcome in univariate analysis. Purposive variable selection was used to select main effects in the final model. All covariates significant at $p<0.10$ were kept in the final model [11].

\section{Time to Death Analysis}

In the time to death analysis, the outcome of interest was date of death based on NDI. For patients who did not die during the study period, they were censored based on: (1) date of last VA healthcare encounter or (2) end of study period (12/31/2014). The survival probability among patients who develop pancreas cancer was compared to patients who did not develop cancer using the Kaplan-Meier method. Univariable Cox proportional hazards regression was used to study the association between each baseline characteristic and time to death. These variables were included as predictors in the multivariable Cox regression model if found moderately associated $(p<0.15)$ with the outcome in univariate analysis. Purposive variable selection was used to select main effects in the final model. All covariates significant at $p<0.10$ were kept in the final model.

\section{Results}

A total of 7211 patients with pancreatic cysts were identified, contributing 31,501 person-years of follow-up (median follow-up 4.4 years). Seventy-nine of 7211 patients (1.1\%) developed pancreas cancer. Thirty-nine of the 79 cancer patients had pathologically confirmed adenocarcinoma; 37 of the 79 patients with pancreatic cancer were identified based on NDI data alone; 3 of the 79 patients were included based on very high clinical suspicion for pancreas cancer, in whom biopsy was never pursued. Pancreas cyst patients who 
developed cancer were older at time of cyst diagnosis compared to patients who never developed cancer (74.1 years vs. 69.2 years; $p<0.01)$. Other demographic characteristics, including gender, race/ethnicity, diabetes, tobacco use, BMI, and CCIS, were similar between individuals with cysts who developed versus did not develop pancreatic cancer on follow-up (Table 1).

Of the 79 patients who developed pancreas cancer on follow-up, the median time to cancer diagnosis after cyst diagnosis was 2.5 years (interquartile range 2.1 years). Sixtyeight patients $(86.1 \%$ of cancer patients, $0.9 \%$ of pancreas cyst cohort) developed cancer 1-5 years after cyst diagnosis; 10 patients $(12.7 \%$ of cancer patients, $0.14 \%$ of pancreas cyst cohort) developed cancer 5-10 years after cyst diagnosis; 1 patient ( $1.3 \%$ of cancer patients, $0.01 \%$ of pancreas cyst cohort) developed cancer more than 10 years after cyst diagnosis. Figure 1 illustrates the cumulative incidence of pancreatic cancer.

Multivariable analysis of predictors of pancreas cancer identified older age (every 1 year; HR 1.05; 95\% C.I. 1.03-1.08) and blacks compared to non-Hispanic whites (HR 1.92; 95\% C.I. 1.07, 3.45) as the only statistically significant variables associated with development of pancreas cancer. Gender, diabetes, tobacco use, BMI, and CCIS were not associated with risk for incident pancreatic cancer on multivariable analysis.

Of the 7211 patients with pancreas cysts in our cohort, 1982 patients $(27.5 \%)$ died during study follow-up. Sixtythree patients died from pancreas cancer $(3.2 \%$ of patient deaths; $0.9 \%$ of pancreas cyst cohort). As expected, patients with pancreas cancer had shorter survival compared to patients who never developed pancreas cancer. Patients who died of any cause during follow-up were older at cyst
Table 1 Demographic and clinical characteristics of pancreas cyst cohort

\begin{tabular}{|c|c|c|c|}
\hline & $\begin{array}{l}\text { Pancreatic cyst patients } \\
\text { with pancreatic cancer }\end{array}$ & $\begin{array}{l}\text { Pancreatic cyst patients } \\
\text { without pancreatic cancer }\end{array}$ & $p$ value \\
\hline Total $N$ & 79 & 7132 & \\
\hline Mean age at cyst diagnosis (SD) & $74.1(10.3)$ & $69.2(11.8)$ & $<0.001$ \\
\hline \multicolumn{4}{|l|}{ Gender, $N(\%)$} \\
\hline Men & $78(98.7 \%)$ & $6780(95.1 \%)$ & \multirow[t]{2}{*}{0.19} \\
\hline Women & $1(1.3 \%)$ & $352(4.9 \%)$ & \\
\hline \multicolumn{4}{|l|}{ Race/ethnicity, $N(\%)$} \\
\hline White & $51(64.6 \%)$ & $5162(72.4 \%)$ & \multirow{3}{*}{0.27} \\
\hline African American & $15(19.0 \%)$ & $1040(14.6 \%)$ & \\
\hline Other & $13(16.5 \%)$ & $930(13.0 \%)$ & \\
\hline Diabetes mellitus, $N(\%)$ & $25(31.6 \%)$ & $2228(31.2 \%)$ & 0.90 \\
\hline \multicolumn{4}{|l|}{ Tobacco use, $N(\%)$} \\
\hline Current smoker & $17(21.5 \%)$ & $1595(22.4 \%)$ & \multirow[t]{4}{*}{0.91} \\
\hline Former smoker & $24(30.4 \%)$ & $1920(26.9 \%)$ & \\
\hline Never smoker & $22(27.8 \%)$ & $2168(30.4 \%)$ & \\
\hline Unknown & $16(20.3 \%)$ & $1449(20.3 \%)$ & \\
\hline \multicolumn{4}{|l|}{ Body mass index, $N(\%)$} \\
\hline$\leq 18.5 \mathrm{~kg} / \mathrm{m}^{2}$ & $1(1.3 \%)$ & $137(1.9 \%)$ & \multirow[t]{7}{*}{0.60} \\
\hline $18.5-25 \mathrm{~kg} / \mathrm{m}^{2}$ & $20(25.3 \%)$ & $1781(25.0 \%)$ & \\
\hline $25-30 \mathrm{~kg} / \mathrm{m}^{2}$ & $25(31.6 \%)$ & $2397(33.6 \%)$ & \\
\hline $30-35 \mathrm{~kg} / \mathrm{m}^{2}$ & $21(26.6 \%)$ & $1339(18.8 \%)$ & \\
\hline $35-40 \mathrm{~kg} / \mathrm{m}^{2}$ & $5(6.3 \%)$ & $445(6.2 \%)$ & \\
\hline$>40 \mathrm{~kg} / \mathrm{m}^{2}$ & $0(0.0 \%)$ & $159(2.2 \%)$ & \\
\hline Unknown & $7(8.9 \%)$ & $874(12.3 \%)$ & \\
\hline \multicolumn{4}{|l|}{ Charlson comorbidity Index score } \\
\hline 0 & $18(22.8 \%)$ & $1715(24.0 \%)$ & \multirow[t]{5}{*}{0.84} \\
\hline 1 & $16(20.3 \%)$ & $1662(23.3 \%)$ & \\
\hline 2 & $14(17.7 \%)$ & $1351(18.9 \%)$ & \\
\hline 3 & $10(12.7 \%)$ & $893(12.5 \%)$ & \\
\hline$\geq 4$ & $21(26.6 \%)$ & $1511(21.2)$ & \\
\hline
\end{tabular}

This table shows the demographic and clinical characteristics of pancreas cyst patients who developed versus did not develop pancreas cancer 


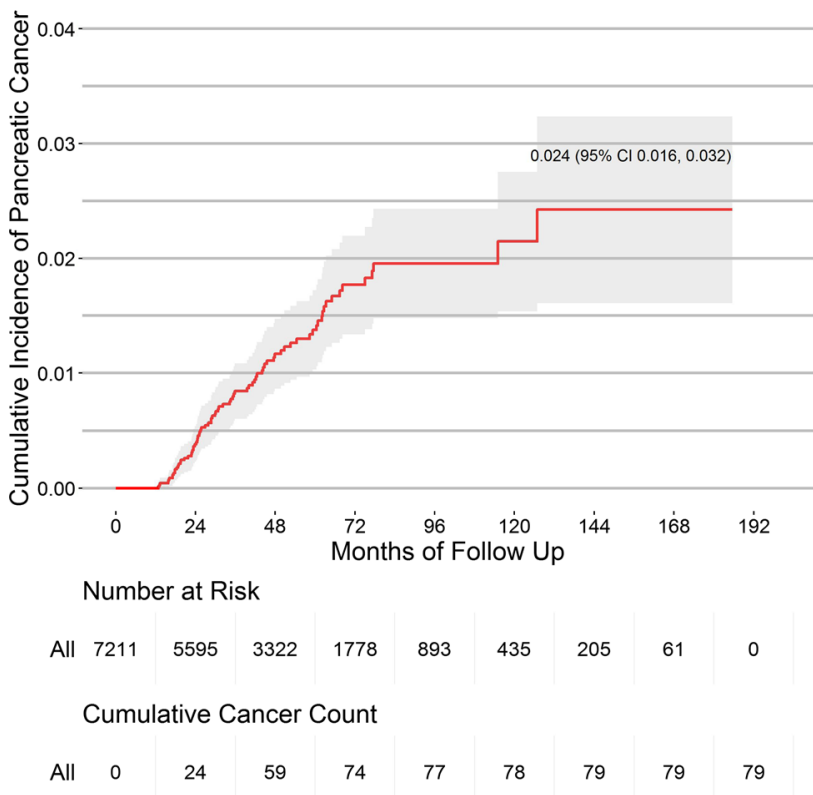

Fig. 1 Cumulative incidence of pancreatic cancer among individuals with pancreatic cysts $(n=7211)$. This figure shows the cumulative incidence of pancreas over time among patients with pancreas cysts utilizing the Kaplan-Meier method. The gray-shaded area represents the $95 \%$ confidence interval

diagnosis (73.7 years vs. 67.6 years; $p<0.01$ ), more frequently men $(97.8 \%$ vs. $94.1 \%, p<0.01)$, more commonly had diabetes (33.8\% vs. $30.3 \%, p<0.01)$, had lower BMI (26.8 vs. $28.4, p<0.01$ ), and had higher mean CCIS at cyst diagnosis ( 2.8 vs. $1.9 ; p<0.01)$ compared to patients who were alive during follow-up. Multivariable analysis identified older age (every 1 year; HR 1.05; 95\% CI 1.04-1.05),

Table 2 Multivariable model of factors associated with all-cause mortality among patients with pancreatic cysts

\begin{tabular}{llr}
\hline Covariate & HR $(95 \%$ CI $)$ & $p$ value \\
\hline Age (every 1 year) & $1.05(1.04,1.05)$ & $<0.001$ \\
Men (Ref = Women) & $1.37(1.00,1.89)$ & 0.053 \\
Tobacco use (Ref = Never Smoker) & & \\
Current smoker & $1.41(1.22,1.63)$ & $<0.001$ \\
Former smoker & $0.98(0.86,1.11)$ & 0.75 \\
Unknown & $1.06(0.92,1.21)$ & 0.43 \\
Body mass index (every 1 kg/m $\left.{ }^{2}\right)$ & $0.96(0.95,0.97)$ & $<0.001$ \\
Charlson comorbidity index score & & \\
(Ref =0) & & \\
CCIS 1 & $1.39(1.17,1.65)$ & $<0.001$ \\
CCIS 2 & $1.74(1.46,2.06)$ & $<0.001$ \\
CCIS 3 & $2.35(1.96,2.81)$ & $<0.001$ \\
CCIS $\geq 4$ & $3.19(2.73,3.74)$ & $<0.001$ \\
\hline
\end{tabular}

Other variables including ethnicity and diabetes were not statistically significant in multivariable analysis male gender (HR 1.37, 95\% CI 1.00-1.89), current smoking (HR 1.41, 95\% CI 1.22-1.63, compared to never smoker), lower BMI (HR 0.96, 95\% CI 0.95-0.97), and higher CCIS at time of cyst diagnosis as significant predictors of death among patients with pancreas cysts (Table 2).

The most common cause of death among all individuals with pancreatic cysts (Fig. 2) was non-pancreas cancer (498 patients; $25.1 \%$ of deaths) followed by cardiovascular disease (398 patients; $20.1 \%$ of deaths). Among the 561 patients with death attributable to cancer, lung cancer was the most common cancer cause of death (137 patients) followed by pancreas ( 63 patients), prostate (51 patients), and colorectal cancer (44 patients).

\section{Discussion}

In this large national cohort of over 7000 US Veterans with 31,501 person-years of follow-up, we found incidence of pancreatic cancer was low, at just $1.1 \%$, corresponding to 2.5 cancers per 1000 person-years follow-up. Further, among patients who develop pancreas cancer, the majority were diagnosed within the first five years of cyst diagnosis, with $14 \%$ developing cancer more than 5 years after pancreas cyst diagnosis. Importantly, among all patients with pancreatic cysts undergoing long-term follow-up, we found pancreas cancer-associated mortality was very low, at just $0.9 \%$, with pancreatic cancer accounting for only $3.2 \%$ of all patient deaths occurring during follow-up. The vast majority of patients who died during follow-up died from non-pancreas cancers (25\%) and cardiovascular causes (20\%).

Our observation of low pancreatic cancer incidence among patients with pancreatic cysts confirms and extends findings from prior population-based studies [8, 12-14].

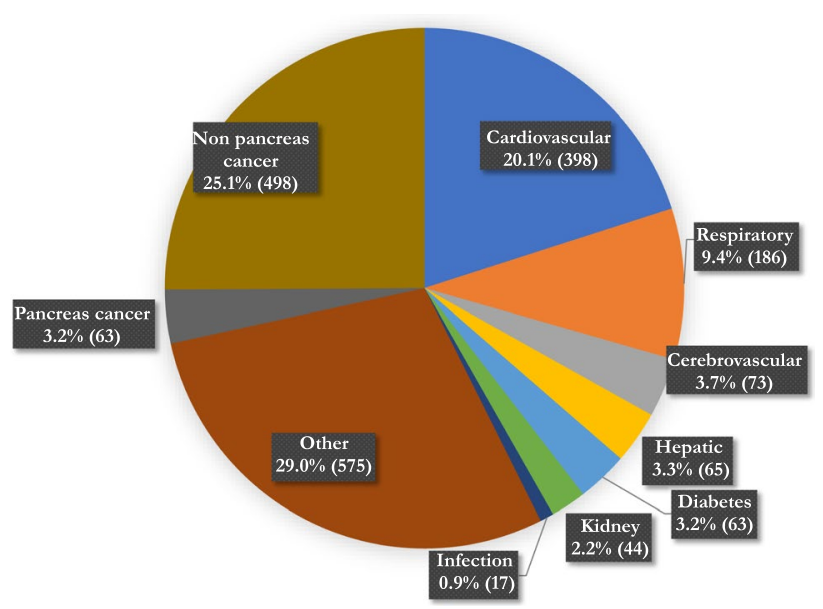

Fig. 2 Causes of death among patients with pancreas cysts; $N=1982$ This figure shows the most common causes of death among patients with pancreas cysts 
Scheiman et al. estimated the lifetime risk of developing pancreas cancer from a pancreas cyst as being less than $1 \%$ when utilizing national US population data and Surveillance, Epidemiology, and End Results (SEER) cancer statistics [8]. Wu et al. evaluated the incidence of pancreas cancer among a surveillance cohort of 1735 patient with pancreas cysts, of whom only $14(0.8 \%$; 4.1 cancers per 1000 person-years follow-up) developed pancreas cancer with average follow-up of 23.4 months (3384 person-years) [13]. The low incidence observed in our study and other population-based studies contrasts with higher pancreatic cancer incidence reported by non-population-based studies. Surgical series usually describe a higher rate of cancers, with a pooled proportion of cysts with invasive cancer closer to $15 \%$ [8]. Applicability of surgical series to clinical decision making is challenged by the limitations created by (a) selection bias, (b) unclear understanding about the long-term outcomes in patients with non-malignant cysts at time of surgery (i.e., would some of these cysts eventually develop cancer?), and (c) presence of substantial proportion of patients with symptomatic pancreas cysts. Other studies (single-center studies, endosonographic databases) also describe higher incidence rates of pancreas cancer $(4-8 \%)[15,16]$. The higher pancreas cancer incidence rates in these studies may be related to referral bias as most of these studies are often from tertiary referral centers and may consist of patients with higher-risk cystic lesions. Other limitations of these studies are small size, small numbers of patients developing pancreatic cancer, and short-term follow-up resulting in imprecise estimates of pancreatic cancer incidence. In contrast to surgical series and other non-population-based studies, our study expands the number of population-based studies confirming that incidence of pancreatic cancer among individuals with pancreatic cysts is low.

Our study also provides new knowledge regarding timing of cancer development following pancreas cyst diagnosis. The American Gastroenterological Association guidelines from 2015 suggest stopping surveillance of pancreas cysts after 5 years if no change in cyst characteristics-a recommendation which was met with criticism [17]. The present study does not specifically address whether the $14 \%$ of patients who developed cancer after 5 years had change in cyst characteristics (such as cyst growth and development of mural nodule) but does demonstrate continued surveillance may be important for a substantial portion of patients with pancreas cysts.

Our study contributes new insights on the mortality after pancreatic cyst diagnosis, an area that has not been widely studied. Kwok et al. examined pancreas cancer-associated mortality among a cohort of patients with pancreas cysts [18]. Of 1800 pancreatic cyst patients, 402 patients (22.3\%) died during a median 5.7-year follow-up. Pancreatic cancerassociated mortality was $2.4 \%(n=43)$, and $11 \%$ of all deaths were due to pancreas cancer. Kwok et al. found a higher pancreas cancer-associated mortality compared to our study ( $2.4 \%$ vs. $0.9 \%$ ), but this may be explained by differences in study design. In the Kwok study, pancreatic cancer was considered preexisting only if diagnosed within 3 months of cyst diagnosis, while our study excluded anyone with pancreas cancer within one year of pancreas cyst diagnosis due to challenges in cancer diagnosis among patient with cystic neoplasms. Median time to cancer diagnosis in the Kwok study for the 43 pancreas cancer deaths was 0.6 years (IQR $0.2,2.1$ ), raising concern some patients had cancer at time of cyst diagnosis. The inclusion of some prevalent cancers likely resulted in higher pancreas cancer-associated mortality compared to our study. Nonetheless, both Kwok et al. and our study support that mortality attributable to pancreatic cancer among patients with pancreatic cysts is low, and much lower that rates of deaths from other causes.

Several limitations may be considered in interpreting our findings. First, this is a retrospective study with inherent limitations. The ideal study design for evaluating the natural history of pancreas cysts would be a prospective cohort study. While such a study would be the gold standard, there are multiple issues with feasibility, cost, and length of follow-up. The outcome of interest, pancreatic cancer, is a rare event, and the size of the study and length of follow-up required would be very costly and inefficient. Second, the current study does not describe cyst-specific characteristics for the cohort of pancreas cyst patients. Due to the size of the cohort and need for manual chart review to extract cystspecific characteristics (such as size, presence of nodule/ solid component, pancreas duct dilation, and cyst growth) obtaining cyst-specific characteristics for the entire cohort is not feasible. Third, another limitation is the use of ICD-9 coding for creation of the pancreas cyst cohort. However, a review of over 400 charts revealed $79.1 \%$ of our cohort met all inclusion criteria. Four, the study is performed among US Veterans and may not be generalizable to other settings. Over 95\% of the pancreas cyst cohort consists of male patients, and certain cystic neoplasms such as MCNs which occur almost exclusively in women are likely under-represented in this cohort. IPMNs, which are felt to be the most common pancreas cyst, likely account for the vast majority of cystic lesions in this study. Our current study has the potential to both underestimate and overestimate pancreas cancer incidence. Pancreas cancer incidence may be underestimated because approximately $20 \%$ of the pancreas cyst cohort did not meet inclusion criteria. However, even if the incidence rate we observed was doubled, the overall estimated incidence of pancreatic cancer would be low. Pancreas cancer incidence may also be overestimated in this study because ICD-9 codes were used to identify patients with pancreas cysts, but it is possible that patients with small cysts were 
not consistently coded, potentially leading to under ascertainment of true cyst diagnosis.

There are several strengths to this study. First, it represents the largest known cohort of pancreas cyst patients with long-term follow-up. Furthermore, this study allows the evaluation of natural history of pancreas cysts with long-term follow-up while minimizing referral bias. Second, compared to other administrative database studies, the use of VINCI allows manual chart review and verification of important diagnoses such as pancreas cancer. Third, there is a robust ascertainment strategy for pancreas cancer diagnosis with use of ICD-9 codes, NDI cause of death data, and cancer registry data. One advantage of using NDI cause of death data is obtaining information regarding patients who may have obtained care from outside of the VA. Furthermore, due to the poor prognosis of pancreas cancer (5-year survival $<9 \%$ ), the vast majority of patients who develop pancreas cancer die within a short period of time and will likely be included within NDI cause of death data. This robust ascertainment strategy ensures high accuracy in identifying pancreas cancer cases.

In conclusion, the overall incidence of pancreas cancer among Veterans with pancreas cysts is low (1.1\%). While most patients develop cancer within the first 5 years of cyst diagnosis, $14 \%$ of patients who developed cancer did so after five years. Pancreas cancer-associated mortality is very low $(0.9 \%)$, and patients are six times more likely to die from a non-pancreas cancer than pancreas cancer. Given incidence of pancreas cancer and death from pancreas cancer are rare, future research should focus on identifying criteria for selecting individuals at high risk for developing and dying from pancreatic cancer.

\footnotetext{
Author's contribution GA: study design, analysis, manuscript writing and review, FY: analysis, manuscript writing and review, LL: study design, analysis, manuscript writing and review, RB: study design, analysis, manuscript writing and review, SSV: analysis, manuscript writing and review, AE: analysis, manuscript writing and review, TS: analysis, manuscript writing and review, SF: analysis, manuscript writing and review, WK: analysis, manuscript writing and review, SG: study design, analysis, manuscript writing and review.
}

Funding 2017 American College of Gastroenterology Clinical Research Award (Anand, PI). "Natural History of Asymptomatic Pancreatic Cysts and Determination of Risk Factors for Development of Pancreatic Cancer Utilizing a Large National Historical Cohort". Grant \# 5 I01 HX001574-05 (Gupta, PI) from the United States Department of Veterans Affairs Health Services Research \& Development Service of the VA Office of Research and Development. The views expressed in this article are those of the authors and do not necessarily represent the views of the Department of Veterans Affairs. Grant \# 5 R37 CA222866-03 (Gupta, PI) from the National Cancer Institute. Grant \# 5 P30 DK 120515-02 (Schnabl, PI) from the National Institute of Diabetes and Digestive and Kidney Diseases.

\section{Declaration}

Conflict of interest The authors have no relevant disclosures related to this manuscript.

Open Access This article is licensed under a Creative Commons Attribution-NonCommercial 4.0 International License, which permits any non-commercial use, sharing, adaptation, distribution and reproduction in any medium or format, as long as you give appropriate credit to the original author(s) and the source, provide a link to the Creative Commons licence, and indicate if changes were made. The images or other third party material in this article are included in the article's Creative Commons licence, unless indicated otherwise in a credit line to the material. If material is not included in the article's Creative Commons licence and your intended use is not permitted by statutory regulation or exceeds the permitted use, you will need to obtain permission directly from the copyright holder. To view a copy of this licence, visit http://creativecommons.org/licenses/by-nc/4.0/.

\section{References}

1. Zanini N, Giordano M, Smerieri E et al. Estimation of the prevalence of asymptomatic pancreatic cysts in the population of San Marino. Pancreatology. 2015;15:417-422

2. Lee KS, Sekhar A, Rofsky NM et al. Prevalence of incidental pancreatic cysts in the adult population on MR imaging. Am J Gastroenterol. 2010;105:2079-2084

3. de Jong K, Nio CY, Hermans JJ et al. High prevalence of pancreatic cysts detected by screening magnetic resonance imaging examinations. Clin Gastroenterol Hepatol. 2010;8:806-811

4. Laffan TA, Horton KM, Klein AP et al. Prevalence of unsuspected pancreatic cysts on MDCT. AJR Am J Roentgenol. 2008;191:802-807

5. de Oliveira PB, Puchnick A, Szejnfeld J et al. Prevalence of incidental pancreatic cysts on 3 tesla magnetic resonance. PLoS One. 2015;10:e 0121317

6. Matsubara S, Tada M, Akahane $\mathrm{M}$ et al. Incidental pancreatic cysts found by magnetic resonance imaging and their relationship with pancreatic cancer. Pancreas. 2012;41:1241-1246

7. Moris M, Bridges MD, Pooley RA et al. Association between advances in high-resolution cross-section imaging technologies and increase in prevalence of pancreatic cysts from 2005 to 2014 . Clin Gastroenterol Hepatol. 2015;14:585-593

8. Scheiman JM, Hwang JH, Moayyedi P. American gastroenterological association technical review on the diagnosis and management of asymptomatic neoplastic pancreatic cysts. Gastroenterology 2015;148:e22

9. Cowper DC, Kubal JD, Maynard C et al. A primer and comparative review of major US mortality databases. Ann Epidemiol. 2002; 12:462-468

10. Management of Diabetes Mellitus Update Working Group. VA/ DoD Clinical Practice Guideline for the Management of Diabetes Mellitus. Version 4.0. Washington DVHAaDo.

11. Hosmer DWLS, May S. Applied survival analysis: regression modeling of time-to-event data, 2nd edn. Hoboken: Wiley-Interscience; 2008.

12. Gardner TB, Glass LM, Smith KD et al. Pancreatic cyst prevalence and the risk of mucin-producing adenocarcinoma in US adults. Am J Gastroenterol. 2013;108:1546-1550

13. Wu BU, Sampath K, Berberian CE et al. Prediction of malignancy in cystic neoplasms of the pancreas: a population-based cohort study. Am J Gastroenterol. 2014;109:121-129 (quiz 130).

14. Kwong WT, Hunt GC, Fehmi SM et al. Low rates of malignancy and mortality in asymptomatic patients with suspected neoplastic 
pancreatic cysts beyond 5 years of surveillance. Clin Gastroenterol Hepatol. 2016;14:865-871

15. Pergolini I, Sahora K, Ferrone CR et al. Long-term risk of pancreatic malignancy in patients with branch duct intraductal papillary mucinous neoplasm in a referral center. Gastroenterology. 2017;153:e1

16. Mukewar SS, Sharma A, Phillip N et al. Risk of pancreatic cancer in patients with pancreatic cysts and family history of pancreatic cancer. Clin Gastroenterol Hepatol. 2018;16:e1

17. Fernandez-Del Castillo C, Tanaka M. Management of pancreatic cysts: the evidence is not here yet. Gastroenterology. 2015; $148: 685-687$
18. Kwok K, Chang J, Duan L et al. Competing risks for mortality in patients with asymptomatic pancreatic cystic neoplasms: implications for clinical management. Am J Gastroenterol. 2017;112:1330-1336

Publisher's Note Springer Nature remains neutral with regard to jurisdictional claims in published maps and institutional affiliations. 